\title{
GISAXS View of Induced Morphological Changes in Nanostructured $\mathrm{CeVO}_{4}$ Thin Films
}

\section{Magdy Lučić Lavčević, ${ }^{1}$ Aleksandra Turković, ${ }^{2}$ Pavo Dubček, ${ }^{2}$ Zorica Crnjak Orel, ${ }^{3}$ Bojan Orel, ${ }^{4}$ and Sigrid Bernstorff ${ }^{5}$}

\author{
${ }^{1}$ Faculty of Chemistry and Technology, University of Split, Teslina 10, 21000 Split, Croatia \\ ${ }^{2}$ Rudjer Boskovic Institute, Bijenička 54, 10000 Zagreb, Croatia \\ ${ }^{3}$ National Institute of Chemistry, Hajdrihova 19, 1000 Ljubljana, Slovenia \\ ${ }^{4}$ Faculty of Computer and Information Science, University of Ljubljana, Trzaska cesta 1001 Ljubljana, Slovenia \\ ${ }^{5}$ Sincrotrone Trieste, Strada Statale 14, km 163,5, in AREA Science Park, 34149 Basovizza, Trieste, Italy
}

Correspondence should be addressed to Magdy Lučić Lavčević, malula@ktf-split.hr

Received 31 May 2010; Revised 11 September 2010; Accepted 30 October 2010

Academic Editor: Quanqin Dai

Copyright (C) 2011 Magdy Lučić Lavčević et al. This is an open access article distributed under the Creative Commons Attribution License, which permits unrestricted use, distribution, and reproduction in any medium, provided the original work is properly cited.

\begin{abstract}
Nanostructured $\mathrm{CeVO}_{4}$ films, designed for applications in electrochemical cells and electrochromic devices, were obtained on glass substrates by the sol-gel process. An analysis of morphological modifications in these films, induced by ultrasonication, annealing, and introduction of lithium ions, was performed, using the grazing-incidence small-angle X-ray scattering technique (GISAXS). The GISAXS results are discussed and related with complementary examinations of the same films in real space, performed by scanning electron microscopy on a different length scale.
\end{abstract}

\section{Introduction}

The performance and integrity of electrodes in advanced electrochemical cells and electrochromic devices are closely related to their morphological characteristics [1]. It was shown in numerous investigations that sol-gel-derived films of titanium, cerium, and vanadium oxides [2-5] exhibit properties, which are desirable for such electrodes. One of the objectives in the research of advanced cells is intercalation of lithium into metal oxide electrodes [6]. The demands on such electrodes are very stringent: they should have significant capacity for lithium ions and maintain their integrity and electrochemical stability over many cycles. Not all demands can be satisfied by a single material. Composing of materials and, additionally, introducing disorder on different length scales (down to nanometric) into the electrode structure can significantly enhance its performance. This approach to improve the design of electrode systems highlights the potential of nanostructured materials that possess large surface areas and short diffusion paths $[7,8]$.
The characterization of sol-gel-derived films of various metal oxides suggests that they are porous nanostructures with a large inner surface area [2-5]. In this paper we focus on sol-gel-derived $\mathrm{CeVO}_{4}$ films, which were prepared with equal molar ratios of vanadium pentoxide $\left(\mathrm{V}_{2} \mathrm{O}_{5}\right)$ and cerium dioxide $\left(\mathrm{CeO}_{2}\right)$, that is, at 55 atomic percent (55 atom \%) of $\mathrm{V}$. As single-material electrodes, transparent $\mathrm{CeO}_{2}$ films show a good capacity for lithium ions and a passive optical behavior under intercalation [9]. $\mathrm{V}_{2} \mathrm{O}_{5}$ films have a layered structure suitable for the intercalation of small ions [1]. As electrodes, they show long-term durability [3]. Previous examinations of film samples, prepared by the sol-gel procedure at different concentrations of $\mathrm{V}$ in $\mathrm{V}_{2} \mathrm{O}_{5} / \mathrm{CeO}_{2}$ films (at 32 atom \%, 38 atom \%, 55 atom \%, and 78 atom \% of V) showed that they exhibit enhanced electrochemical properties and stability in comparison to standard oxide films [10]. These improvements indicated that nanostructured, mixed $\mathrm{V}_{2} \mathrm{O}_{5} / \mathrm{CeO}_{2}$ films, especially those prepared at 55 atom $\%$ of $\mathrm{V}$ (i.e., $\mathrm{CeVO}_{4}$ films) were structurally well designed for lithium intercalation. 
Generally, nanostructured sol-gel-derived films can be considered as two-phased systems containing nanosized particles and pores. This model, originally suggested by small-angle X-ray scattering (SAXS) analysis, was also confirmed by complementary investigations performed on films with electron microscopy and X-ray diffraction. Particularly, in case of $\mathrm{TiO}_{2}$, the estimation of the average crystallite sizes with the Debye-Scherrer' method in X-ray diffraction analysis was in accordance with the estimation of the average particle sizes using Guinier's method in SAXS analysis [2]. Our previous SAXS-based morphological analysis of $\mathrm{CeVO}_{4}$ films, derived by a standard sol-gel procedure, showed that they could also be considered as systems built of nanosized particles which are distributed throughout the films and which are growing during annealing [5].

As a continuation of the search for the best division of metal oxides in order to improve their electrochemical performance, the present analysis reports on the morphological modifications which can eventually be induced in $\mathrm{CeVO}_{4}$ oxide films by ultrasonication, annealing, and introduction of lithium ions. The analysis is based on the use of the two-dimensional grazing-incidence X-ray scattering (2D GISAXS) technique. In this technique, the penetration of Xrays in the film can be controlled by choosing an appropriate grazing angle of incidence. Our measurements were performed with a grazing angle of incidence close to the critical angle of total reflection for $\mathrm{CeVO}_{4}$ films. The SAXS analysis is generally suitable for the estimation of the size of scattering objects; the 2D detection of the scattering patterns enables an additional insight into the films' morphology, including eventual anisotropy and correlations effects. However, the interpretation of GISAXS features can be very ambiguous, especially in systems like ours, and the complete morphology description cannot be reached solely by 2D GISAXS analysis. Therefore the nanostructures were investigated also in real space with scanning electron microscopy.

\section{Experimental}

$\mathrm{CeVO}_{4}$ films were obtained via the inorganic sol-gel process at 55 atom \% of $\mathrm{V}$ [10]. The preparation of an aqueous dispersion of hydrated oxide (sol) $\mathrm{V}_{2} \mathrm{O}_{5}, \mathrm{CeO}_{2}$, and mixed $\mathrm{V}_{2} \mathrm{O}_{5} / \mathrm{CeO}_{2}$ started from a solution of the corresponding metal oxide or metal salt, $\mathrm{V}_{2} \mathrm{O}_{5}$, and $\mathrm{Ce}\left(\mathrm{NH}_{4}\right)_{2}\left(\mathrm{NO}_{3}\right)_{6}$, respectively. Crystalline $\mathrm{V}_{2} \mathrm{O}_{5}$ was dissolved in $6 \%$ nitric acid to obtain $0.044 \mathrm{M}$ aqueous solution. By the evaporation of water, the desired concentration of $\mathrm{V}_{2} \mathrm{O}_{5}$ was obtained. From the aqueous solution of $\mathrm{Ce}\left(\mathrm{NH}_{4}\right)_{2}\left(\mathrm{NO}_{3}\right)_{6}$ precipitates were obtained by addition of $\mathrm{NH}_{4} \mathrm{OH}$ until $\mathrm{pH}=9$ was reached. The peptization was performed by the addition of equimolar quantity of $\mathrm{HNO}_{3}$, aged up to $363 \mathrm{~K}$. By diluting in water, the $\mathrm{CeO}_{2}$ sol of the desired concentration was obtained. Mixed sol was obtained by adding 55 atom $\%$ of $\mathrm{CeO}_{2}$ sol into the $\mathrm{V}_{2} \mathrm{O}_{5}$ sol. As a new step in the preparation procedure, high energy and high frequency sound $(125 \mathrm{~W}, 20.1 \mathrm{kHz})$ were applied to the sol for 10 minutes. The films were deposited on $\mathrm{SnO}_{2}$ :F glass substrates $\left(2 \times 3 \times 0.1 \mathrm{~cm}^{3}\right)$ by the dipcoating technique, as one-dip layers, with pulling speed of $10 \mathrm{~cm} / \mathrm{min}$, and heated at $673 \mathrm{~K}$.
Four samples were prepared: sample A1, prepared as described and annealed for 5 minutes; sample A2, prepared as described and annealed for 15 minutes; sample B1, prepared as A1 and intercalated with lithium ions; sample $\mathrm{B} 2$, prepared as $\mathrm{A} 2$ and intercalated with lithium ions.

Scattering experiments were performed at the SAXS beamline [11], at the ELETTRA synchrotron radiation source in Trieste (Italy). A photon energy of $8 \mathrm{keV}(\lambda=$ $0.155 \mathrm{~nm}$ ) was used, and the beam size was $4 \mathrm{~mm} \times 0.150 \mathrm{~mm}$ (horizontal $\times$ vertical). The film samples on glass substrates were examined, using grazing-incidence geometry [12]. For $\mathrm{X}$-rays, the index of refraction, $n$, of solids is less than one, that is, $n=1-\eta^{\prime}-i \eta^{\prime \prime}$, where $\eta^{\prime}$ and $\eta^{\prime \prime}$ are the real (dispersion) and imaginary (absorption) parts of the correction term, respectively. For small incident angles of X-rays, measured with respect to the film surface, it is convenient to introduce the so-called critical angle, $\alpha_{c}=\sqrt{ } 2 \eta^{\prime}$. Namely, at angles smaller than $\alpha_{c}$, total reflection of X-rays takes place. In grazing-incidence geometry, the grazing-incident angle, $\alpha_{i}$, is close to $\alpha_{c}$. For grazing-incident angles larger than the critical, the reflectivity decreases steeper than the value given by the Fresnel theory because of deviations of the sample surface from ideal flatness. In the case of our samples, the measurements were performed at grazing-incident angles slightly larger than the critical angle, providing a penetration depth up to $100 \mathrm{~nm}$ [13]. The critical angle (around $0,37^{\circ}$ ) was determined experimentally for each film.

In the used setup for GISAXS measurements, samples were mounted on a stepping-motor-controlled tilting stage with a step resolution of $0.001^{\circ}$. At first, the stage (and the sample surface) was aligned horizontally and parallel to the incoming beam within $\pm 0.1^{\circ}$. The scattering geometry is shown in Figure 1. The sample orientation with respect to the incident beam, specified by the wave vector $\mathbf{k}_{\mathbf{i}}$, was chosen in order to keep the grazing-incident angle in the range $0.4^{\circ}<\alpha_{i}<1.4^{\circ}$, for which the effective area of the beam foot print is smaller than the sample surface area $(20 \mathrm{~mm} \times 20 \mathrm{~mm})$. The outgoing beam (with the exit angle $\alpha_{f}$ ) is presented by the wave vector $\mathbf{k}_{\mathbf{f}}$. The maximum of the scattered intensity is in the direction of the specular reflection, $\mathbf{k}_{\mathrm{SR}}$. For the specularly reflected beam, the exit angle equals the incident angle. Together with the reflected beam, a diffuse scattering is present, although of much lower intensity. This diffuse scattering from a surface, measured under nonspecular condition, that is, when the exit angle is different from the incident angle, yields information about structural features along the surface. This diffuse surface scattering contribution is concentrated near the specular plane. When well-defined particles are randomly distributed within the film, they cause additional diffuse scattering. This type of scattering can be easily separated from the surface scattering, since it does not depend on the incident angle. Also, it is easily recognized, since its contribution is a function of the total scattering angle $(2 \theta)$ only and is constant for $|\mathbf{q}|^{2} \approx q_{y}^{2}+q_{z}^{2}=$ constant, where $\mathbf{q}=\mathbf{k}_{\mathbf{f}}-\mathbf{k}_{\mathbf{i}}$ is the total scattering wave vector (Figure 1). The absolute value of the scattering wave vector equals $q=2 \pi \sin \theta / \lambda$.

The scattering intensity spectra were recorded by a $2 \mathrm{D}$ X-ray CCD detector, containing $1024 \times 1024$ pixels and 


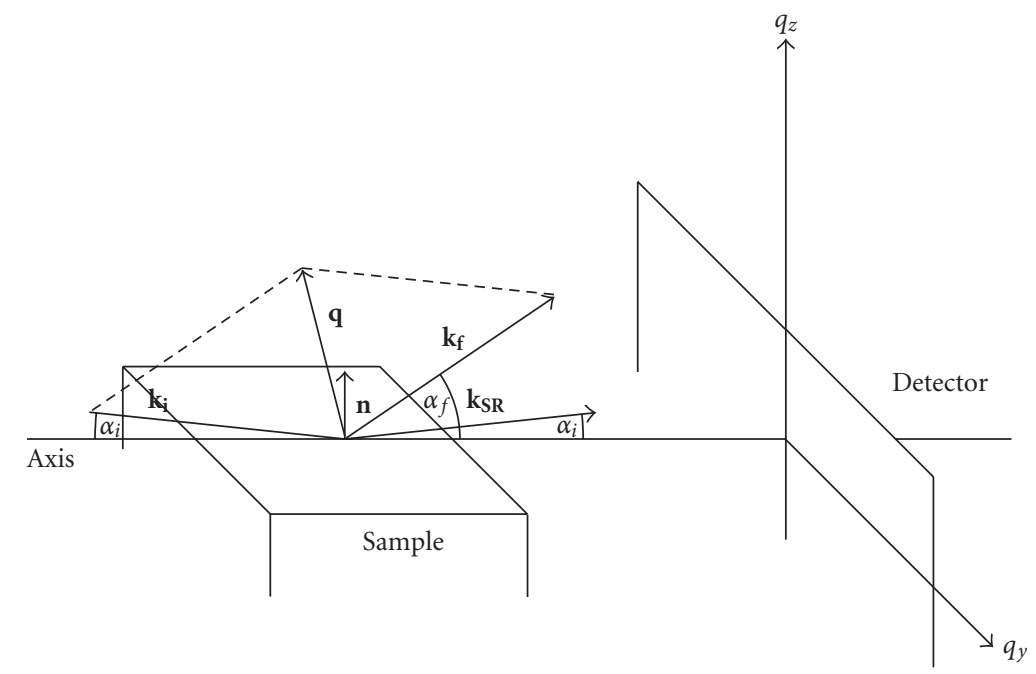

FIgURE 1: The geometry of GISAXS measurements. $\mathbf{k}_{\mathbf{i}}$ is the wave vector of the incident X-ray beam, $\mathbf{k}_{\mathbf{f}}$ is the wave vector of the outgoing $\mathrm{X}$-ray beam, and $\mathbf{q}$ is the scattering wave vector, with the components, $q_{y}$ and $q_{z}$. The unit vector $\mathbf{n}$ is normal to the sample surface. The wave vector $\mathbf{k}_{\mathbf{S R}}$ is in the direction of the specular reflection.

positioned perpendicular to the incident beam. A thin $\mathrm{Al}$ strip was placed in front of the detector to avoid its overflow in the specular plane direction, where the usually much stronger surface (specular and diffuse) scattering is present and in order to have better resolution of the diffuse part of the scattering off the specular plane (particle contribution). The recorded spectra were corrected for readout noise and detector response.

Samples were finally analyzed by field emission scanning electron microscopy (FSEM).

\section{Results and Discussion}

2D GISAXS intensity maps, recorded for samples A1, A2, B1 and B2 are shown in Figures 2(a), 2(b), 2(c), and 2(d). The maximum of the scattered intensity is in the direction of the specular plane $\left(q_{y}=0\right)$, which is normal to the surface of the sample. This scattered intensity is partly reduced by a thin $\mathrm{Al}$ absorber (vertical strips in the patterns are depleted intensities). The lower part of the scattering is missing because of the absorption in the sample. For exit angles equal to incident critical angle $\left(\alpha_{f}=\alpha_{c}\right)$, there is an enhancement in the scattering intensity due to the refraction effects, which is also known as Yoneda peak.

By comparing the patterns of samples A1 and A2, we see that a change in morphology is induced by prolonged annealing. Furthermore, the differences in the patterns of $\mathrm{A} 1$ and $\mathrm{B} 1$ indicate that the change in morphology of the initial sample A1 also takes place as a consequence of the intercalation of lithium. There is no qualitative essential difference between patterns of samples A2 and B2, so it seems that intercalation in a prolongedly heated sample does not strongly affect its morphology on the nanoscale.

Besides the analysis of features of the 2D GISAXS maps, the method of interpreting scattering data is based on the analysis of scattering curves, which show the dependence of the intensity, $I$, on the scattering vector, $\mathbf{q}$. We extracted 1D scattering curves from the 2D GISAXS intensity maps as vertical (parallel to the specular plane) and horizontal (parallel to the sample surface) scans (or cuts) of scattering intensity versus $q_{z}$ and $q_{y}$, respectively. The unique feature exhibited by sample A1, a maximum at a higher $q$-value in the specular (vertical) direction, is also seen in Figure 3. In this figure the scattering curves of sample A1 are displayed, extracted as vertical cuts from $2 \mathrm{D}$ patterns at a fixed horizontal angle, for three different grazing-incident angles of the X-rays. The position of the peak does not change with the increment of the incident angle, but its intensity grows with the penetration depth. A peak with such characteristics is usually exhibited by systems of very concentrated or strongly correlated particles. In cases of homogenous particles it corresponds to the average distance between particles. Assuming that the position of the peak corresponds to the most prominent length in the vertical direction, we can estimate the value of this length, $L\left(q_{z}\right)=2 \pi / q_{z}^{\text {peak }}=3.5 \mathrm{~nm}$, where $q_{z}^{\text {peak }}$ is the position of the maximum of the scattered intensity in $q$-space. (This estimation is not exact-it serves as a rough estimate in characterizing the trends of changes in the morphology.) A possible explanation is that this peak represents a contribution of scattering objects with a rather low dispersion of sizes. With increasing polydispersity, such contribution should rapidly drop. Consequently, from the absence of such a peak in the scattering patterns of samples $\mathrm{A} 2$ and $\mathrm{B} 1$, it can be concluded that the scattering objects under prolonged annealing as well as under intercalation become polydispersed, disordered systems. However, while the absence of the interference peak is the essential qualitative difference between the patterns of samples $\mathrm{A} 1$ and $\mathrm{A} 2 / \mathrm{B} 2$, the GISAXS map and the information extracted from the scattering curves of sample B1 indicate that the intercalation process brings up a more drastic change. The intensity scans, taken from Figure 2 at fixed horizontal and vertical angle 


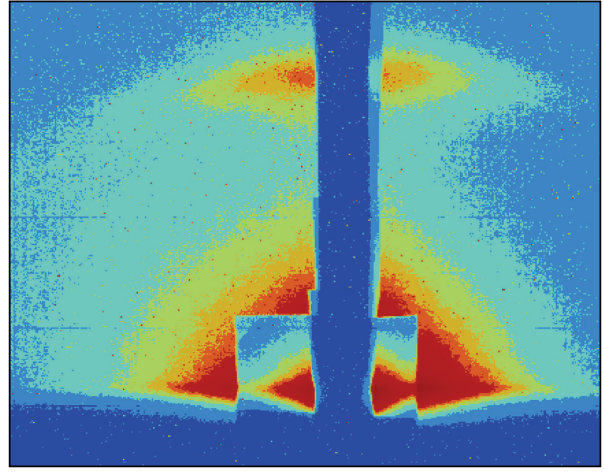

(a)

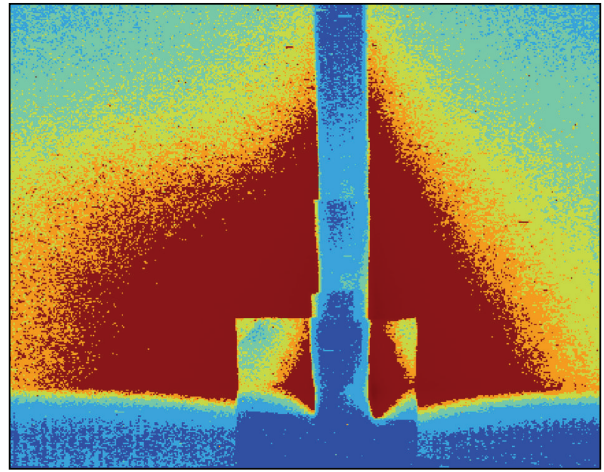

(c)

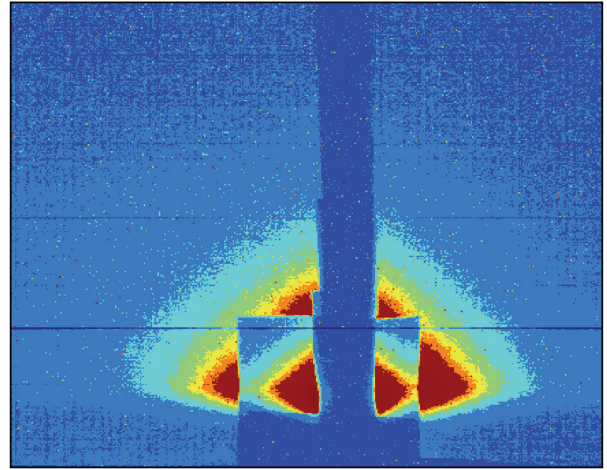

(b)

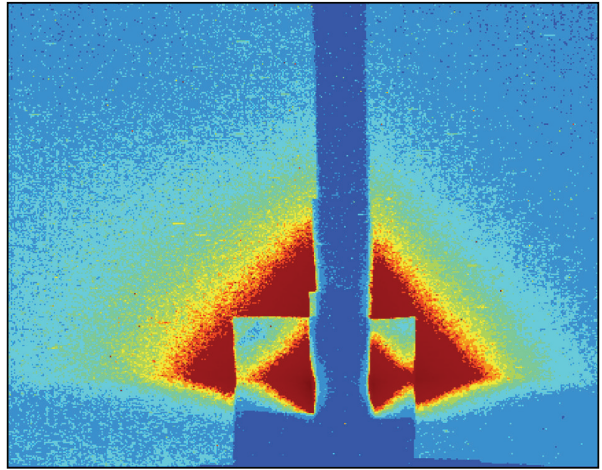

(d)

Figure 2: 2D GISAXS patterns of (a) sample A1, (b) sample A2, (c) sample B1, and (d) sample B2.

(relatively close to the specular plane and close to the Yoneda peak, resp.), were used to calculate another morphological parameter, the Guinier radius, $R_{G}$, which can be used for the estimation of the average particle size.

In the Guinier approximation, scattering in the small $q$ region depends only on the overall size of the particle and not on its shape, so the scattered intensity $I(q)$ can be expressed as

$$
I(q)=C_{G} \exp \left(-\frac{R_{G}^{2} q^{2}}{3}\right) .
$$

The Guinier radius is the average radius of gyration of a dilute set of randomly oriented scattering centers. When this condition is not fulfilled, it still can be calculated as a rough approximation. We determined the radii of gyration from the scattering curves, extracted from the GISAXS patterns of Figure 2 as vertical and horizontal intensity cuts, by plotting the curves in the $\operatorname{Ln} I$ versus $q^{2}$ presentation and fitting each of them by a line for very small wave vectors. The fitting of the scattering curves, that is, of a vertical and a horizontal cut, is represented in Figure 4 for the sample A2.

For a slope $\beta$ of the line, the corresponding Guinier gyration radius is calculated as

$$
R_{G}=(-3 \beta)^{1 / 2}
$$

The determined slopes and gyration radii for the samples A1, A2, B1, and B2 are shown in Table 1.
TABLe 1: The slopes of the fitting lines $(\beta)$ and Guinier radii $\left(R_{G}\right)$ for samples A1, A2, B1, and B2, calculated from vertical and horizontal intensity cuts. $L\left(q_{z}\right)$ is the prominent length, estimated for sample A1.

\begin{tabular}{lccccc}
\hline Sample & & $\mathrm{A} 1$ & $\mathrm{~A} 2$ & $\mathrm{~B} 1$ & $\mathrm{~B} 2$ \\
\hline \multirow{2}{*}{ Vertical cut } & $\beta$ & -1.1 & -3.2 & -1.1 & -2.4 \\
& $R_{G} / \mathrm{nm}$ & $(1.8)$ & 3.1 & 1.8 & 2.7 \\
\multirow{2}{*}{ Horizontal cut } & $\beta$ & -3.2 & -8.3 & $-1.46 /-96$ & -6.2 \\
& $R_{G} / \mathrm{nm}$ & $(3.1)$ & 5.0 & $2.1 / 17$ & 4.3 \\
Vertical cut & $L\left(q_{z}\right) / \mathrm{nm}$ & 3.5 & $/$ & $/$ & $/$ \\
\hline
\end{tabular}

The values of $R_{G}$ for sample A1 (in parentheses in Table 1), should be taken with caution in further consideration, because the Guinier approximation is generally not valid for the description of particles systems revealing an interference effect. In such case, the most prominent length, $L$, is the most relevant value.

We believe that in sample A1, as a consequence of ultrasonication, a formation of small particles takes place, which are stacked vertically on each other. The value of $R_{G}$ in vertical direction roughly corresponds to the estimated prominent length in that direction, $L\left(q_{z}\right)$. Any possible correlation in the horizontal direction is not seen by GISAXS with the used setup. If particles were horizontally correlated (with $L\left(q_{y}\right)$ similar to $L\left(q_{z}\right)$ ), the correlation peak would be 


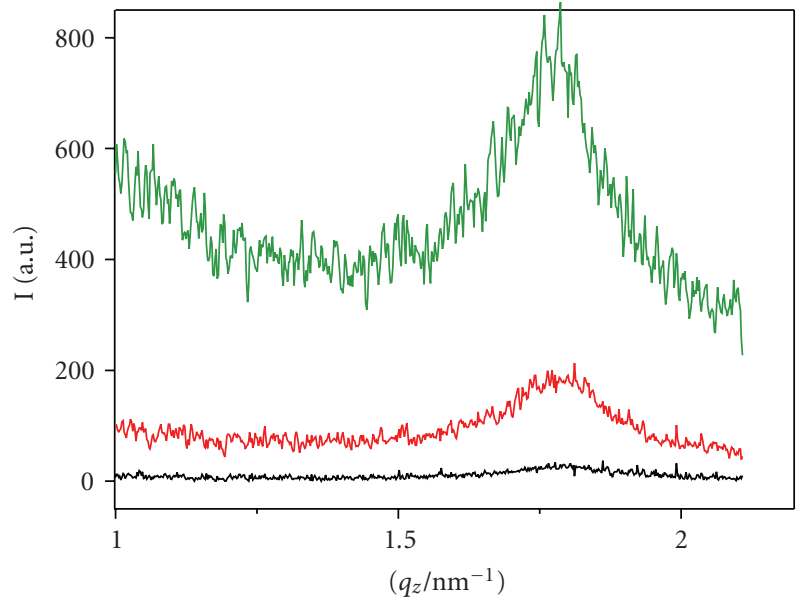

Figure 3: Scattering curves of sample A1, extracted as vertical cuts at a constant horizontal angle, showing a peak at $q_{z}=1.8 \mathrm{~nm}^{-1}$ for three incident grazing angles of X-rays: below the critical angle (black) and above the critical angle (red and green).

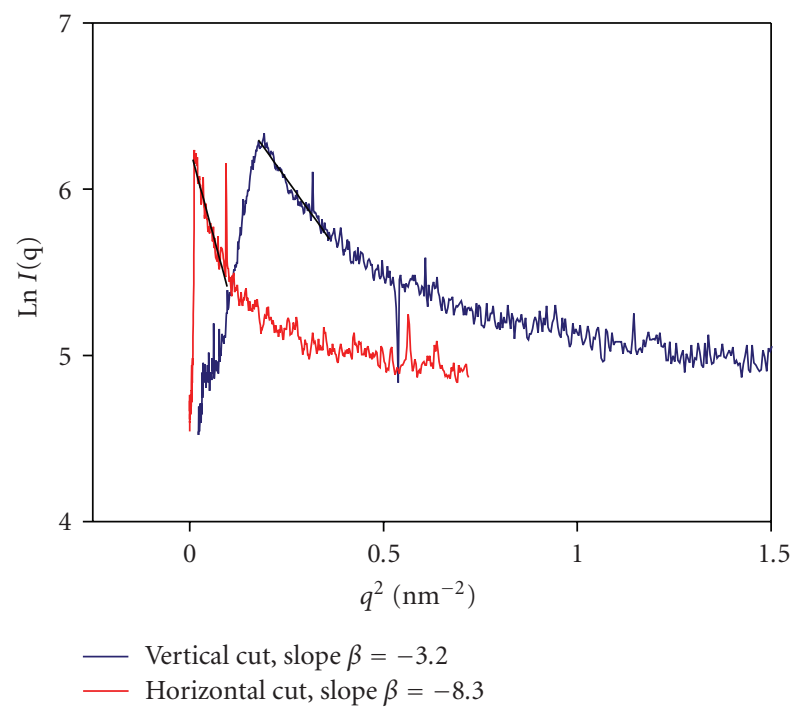

Figure 4: Scattering curves of sample A2, extracted as a vertical cut at a constant horizontal angle (blue) and a horizontal cut at a constant vertical angle (red), shown in the presentation suitable for determination of the average gyration radius. $\beta$ is the slope of the fitting lines (black).

positioned beyond the experimental $q_{y}$ range. However, the value of $R_{G}$ in horizontal direction $(3.1 \mathrm{~nm})$ suggests either aggregation or elongation of particles in the horizontal plane. The FSEM picture of the sample A1 (Figure 5(a)) shows a uniformly structured surface with small grains, and thus supports the existence of the presumed morphology on the nano scale.

The analysis of horizontal scans of sample B1 showed the existence of at least two size distributions of particles, with a significant difference in their average radii. The smaller particles are almost spherical (with an average diameter of around $4 \mathrm{~nm}$ ), because the values of $R_{G}$ are similar in both directions. Again, the FSEM picture of this sample (Figure 5(c)) can be correlated to the GISAXS view. Obviously, it shows a morphology transformation induced by intercalation of lithium, which causes the lack of its overall homogeneity. It seems that intercalation acts as a trigger for formation of particles with a very broad size distribution which extends over a decade. In such case, vertical cuts are not appropriate for the gyration radius analysis (only small particles are detected). The limited possibilities in the GISAXS detection can also affect the results - the contribution of large particles can be hidden by the beamstop. This, complex morphology of sample B1 explains the development of a diffuse, featureless signal of the GISAXS pattern of this sample (Figure 2(c)).

The gyration radii obtained from a horizontal and a vertical intensity scan, for samples A2 and B2, have different sizes (Table 1), which indicate the anisotropy of the particles. Anisotropy in SAXS results from more or less oriented anisotropic particles. Although Guinier approximation gives only the radius as a measure of particle size, it is also partly sensitive to oriented flat or elongated particles. The radius is obtained from integrating the contribution to scattering over the particle volume and possible orientations. A more complex model, with assumed particle shape, as well as variations of the particle extension in different directions would reproduce the measured scattering more precisely. However, further experimental investigation would be necessary in order to determine some of these variations, or the result of the fitting would be merely a numerical one. The applied, simplest model gives information about the sample anisotropy without unnecessarily relying on numerical calculus.

In conclusion, the above GISAXS results, together with the FSEM scans, are understood as follows: a starting configuration of sample A1, which includes vertically correlated particles, gets changed by postpreparation treatments. Under prolonged annealing (sample A2), particles grow bigger and the order is lost. Lithium intercalation drastically changes the starting configuration of sample A1. The sample B1 consists of a mix of particles, with distribution of sizes (and shapes), which escape any simple description. Obviously, the ordered configuration of sample A1 is not stable enough to be suitable for lithiation. On the other hand, lithiation of the prolongedly heated sample does not abruptly affect the morphology on the nanoscale-samples A2 and B2 exhibit similar parameters. In comparison with sample A2, sample B2 shows a slight reduction of the average gyration radii in both directions (a redistribution of particle sizes takes place in the A2 to B2 transition). So, although the morphology is affected by lithiation, the similarity of the 2D GISAXS patterns and particle sizes suggests that the type of morphology in samples A2 and B2 is the same. A slight smoothing of the surface can be detected by comparing the corresponding FSEM pictures of samples A2 and B2 (Figures 5(b), and $5(d)$ ), which reflect the reduction in the average particle size.

Further investigation of the electrochemical and optical properties and of the stability of films should clarify the correlation between the determined morphological variations and the performance of films as electrodes. 


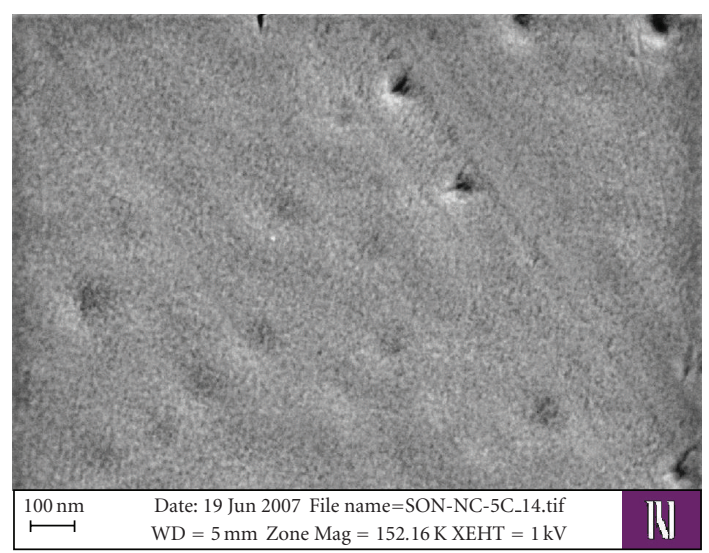

(a)

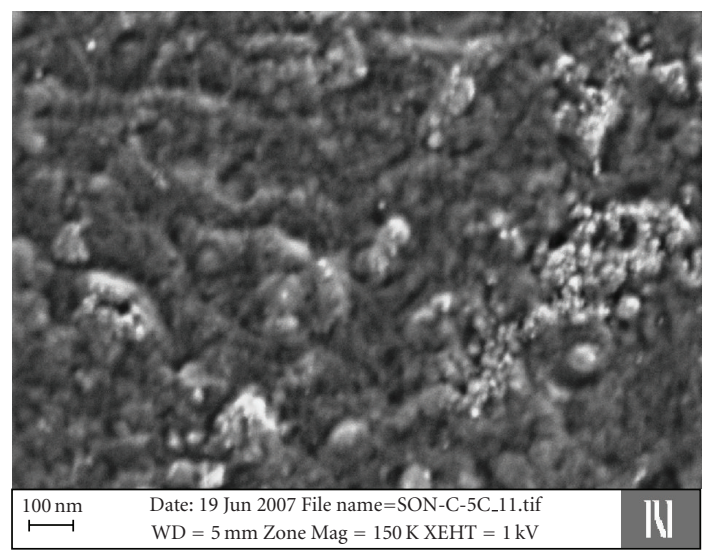

(c)

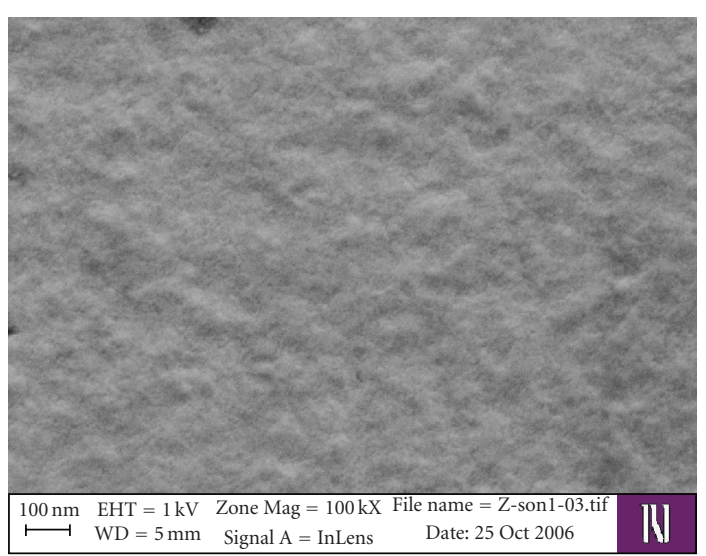

(b)

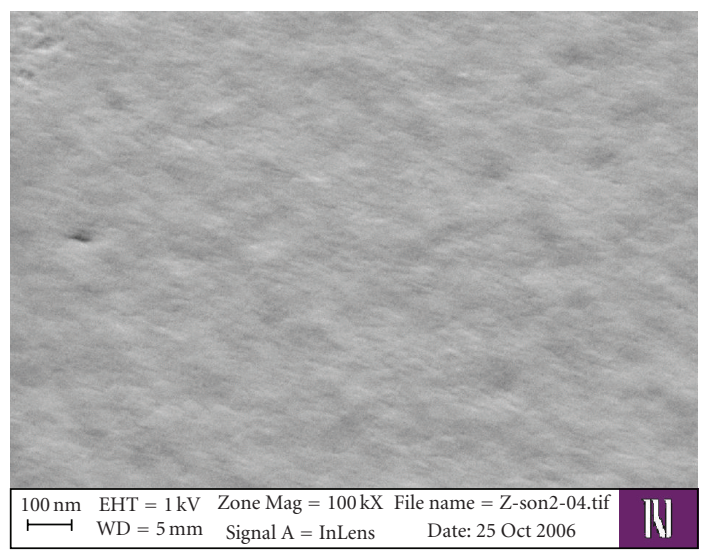

(d)

Figure 5: FSEM pictures of (a) sample A1, (b) sample A2, (c) sample B1, and (d) sample B2.

\section{Acknowledgment}

The results shown in this work are obtained in the frame of the scientific project "Nanostructures of metal oxides and metals: morphology and properties," supported by the Ministry of Science, Education and Sport of the Republic of Croatia.

\section{References}

[1] A. Talledo, A. M. Andersson, and C. G. Granqvist, "Structure and optical absorption of LiVO thin films," Journal of Applied Physics, vol. 69, no. 5, pp. 3261-3265, 1991.

[2] M. L. Lavčević and A. Turković, "Small-angle X-ray scattering and wide-angle X-ray diffraction on thermally annealed nanostructured TiO films," Thin Solid Films, vol. 419, no. 12, pp. 105-113, 2002.

[3] F. Zhang, S. Passerini, B. B. Owens, and W. H. Smyrl, "Nanocomposites of $\mathrm{VO}$ aerogel and $\mathrm{RuO}$ as cathode materials for lithium intercalation," Electrochemical and Solid-State Letters, vol. 4, no. 12, pp. A221-A223, 2001.

[4] M. L. Lavčević, A. Turković, P. Dubček, and S. Bernstorff, "Nanostructured CeO thin films: a SAXS study of the interface between grains and pores," Thin Solid Films, vol. 515, no. 14, pp. 5624-5626, 2007.
[5] M. L. Lavčević, P. Dubček, A. Turković, Z. Crnjak-Orel, and S. Bernstorff, "Nanostructural depth profile of vanadium/cerium oxide film as a host for lithium ions," Solar Energy Materials and Solar Cells, vol. 91, no. 7, pp. 616-620, 2007.

[6] M. Winter, J. O. Besenhard, M. E. Spahr, and P. Novák, "Insertion electrode materials for rechargeable lithium batteries," Advanced Materials, vol. 10, no. 10, pp. 725-763, 1998.

[7] C. R. Sides, N. Li, C. J. Patrissi, B. Scrosati, and C. R. Martin, "Nanoscale materials for lithium-ion batteries," MRS Bulletin, vol. 27, no. 8, pp. 604-596, 2002.

[8] A. S. Aricò, P. Bruce, B. Scrosati, J. M. Tarascon, and W. Van Schalkwijk, "Nanostructured materials for advanced energy conversion and storage devices," Nature Materials, vol. 4, no. 5, pp. 366-377, 2005.

[9] M. Veszelei, L. Kullman, A. Azens, C. G. Granqvist, and B. Hjörvarsson, "Transparent ion intercalation films of $\mathrm{Zr}-\mathrm{Ce}$ oxide," Journal of Applied Physics, vol. 81, no. 4, pp. 2024-2026, 1997.

[10] Z. C. Orel, "New counter electrode prepared as vanadium oxide and V/Ce oxide films: preparation and characterization," Solid State Ionics, vol. 116, no. 1-2, pp. 105-116, 1999.

[11] H. Amenitsch, S. Bernstorff, and P. Laggner, "High-flux beamline for small-angle x-ray scattering at ELETTRA," Review of Scientific Instruments, vol. 66, no. 2, pp. 1624-1626, 1995. 
[12] J. R. Levine, J. B. Cohen, Y. W. Chung, and P. Georgopoulos, "Grazing-incidence small-angle X-ray scattering: new tool for studying thin film growth," Journal of Applied Crystallography, vol. 22, no. 6, pp. 528-532, 1989.

[13] P. Dubček, "Nanostructure as seen by the SAXS," Vacuum, vol. 80, pp. 92-97, 2005. 

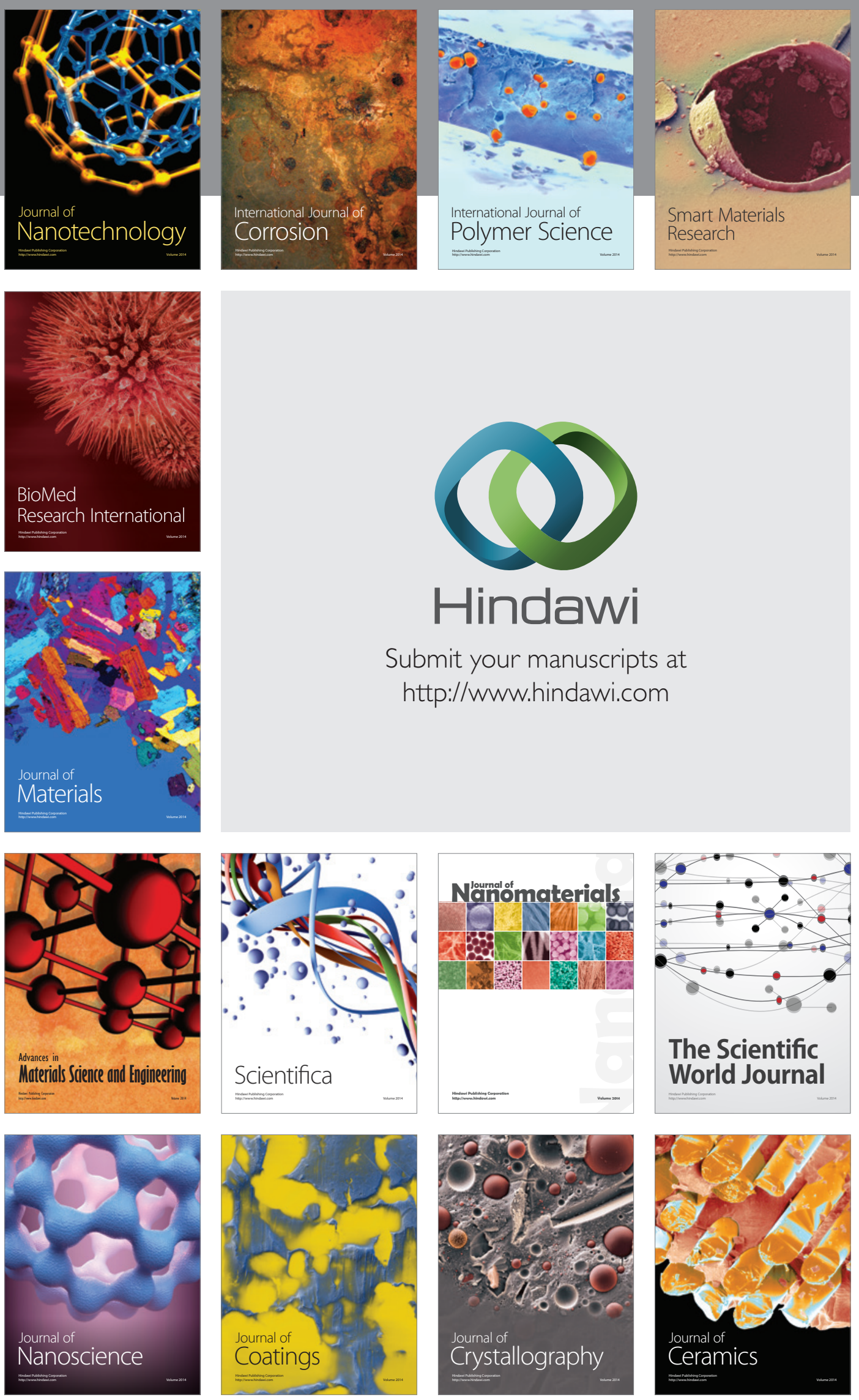

The Scientific World Journal

Submit your manuscripts at

http://www.hindawi.com

\section{World Journal}

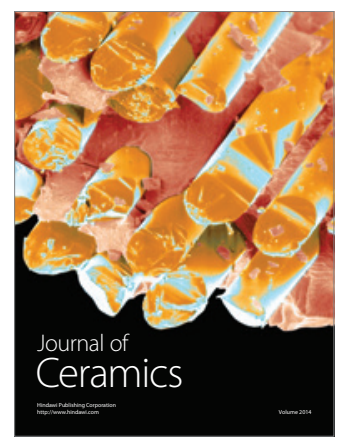

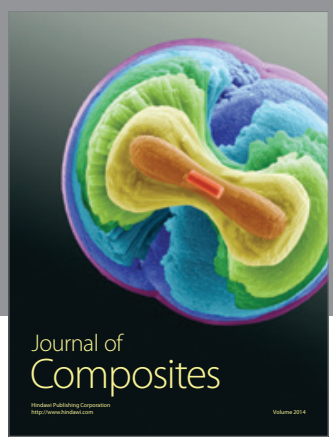
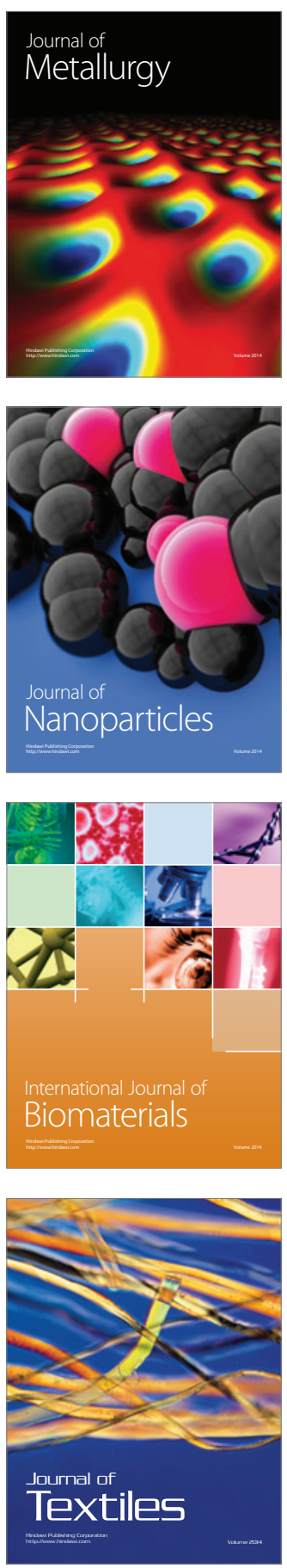